University of Nebraska - Lincoln

DigitalCommons@University of Nebraska - Lincoln

Faculty Publications, Department of Physics and Astronomy

Research Papers in Physics and Astronomy

6-10-2005

\title{
Comment on "Fano Line Shapes Reconsidered: Symmetric Photoionization Peaks from Pure Continuum Excitation"
}

John W. Cooper

Institute for Physical Science and Technology, University of Maryland

Chris H. Greene

University of Colorado, Boulder, chgreene@purdue.edu

Peter W. Langhoff

San Diego Supercomputer Center, University of California--La Jolla

Anthony F. Starace

University of Nebraska-Lincoln, astarace1@unl.edu

Carl Winstead

A. A. Noyes Laboratory of Chemical Physics, California Institute of Technology

Follow this and additional works at: https://digitalcommons.unl.edu/physicsfacpub

Part of the Physics Commons

Cooper, John W.; Greene, Chris H.; Langhoff, Peter W.; Starace, Anthony F.; and Winstead, Carl, "Comment on "Fano Line Shapes Reconsidered: Symmetric Photoionization Peaks from Pure Continuum Excitation"'" (2005). Faculty Publications, Department of Physics and Astronomy. 38.

https://digitalcommons.unl.edu/physicsfacpub/38

This Article is brought to you for free and open access by the Research Papers in Physics and Astronomy at DigitalCommons@University of Nebraska - Lincoln. It has been accepted for inclusion in Faculty Publications, Department of Physics and Astronomy by an authorized administrator of DigitalCommons@University of Nebraska Lincoln. 
Comment on "Fano Line Shapes Reconsidered: Symmetric Photoionization Peaks from Pure Continuum Excitation"

Eichmann, Gallagher, and Konik recently reported [1] an experimental study of electronic autoionization that they interpret as revealing "...a shortcoming of the straightforward application of Fano's theory...," referring to the well-known 1961 publication of the late Ugo Fano [2], and further suggested that his theory "...fails to describe photoionization...". In this Comment we indicate that Fano's 1961 treatment of photoionization is capable of explaining the general appearance of the observed spectrum entirely within the context of the interpretive assumptions and particular basis-state designations adopted by these authors [1].

The experiment employs a four-laser Stark-switching preparation technique to populate doubly excited $(5 d ; n=$ $17, l=12)$ planetary states of $\mathrm{Sr}$ atoms, where $5 d$ and $n, l$ are independent-particle quantum numbers for the (nonoverlapping) inner Rydberg and outer hydrogenic electrons, respectively, [1]. A fifth laser excites to a series of $\left(5 g ; n l^{\prime}\right)$ states that autoionize into the $(5 f ; \epsilon l)$ continuum.

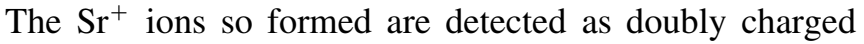
$\mathrm{Sr}^{++}$ions after absorbing a second photon from the fifth laser. It is clear that both the discrete $\left(5 d ; 17,12 \rightarrow 5 g ; n l^{\prime}\right)$ and continuum $(5 d ; 17,12 \rightarrow 5 f ; \epsilon l)$ excitations are dipole forbidden in the independent-particle basis of [1] (and consequently, Fano's $q$ parameter is indeterminate, i.e., $0 / 0$ ). Nonetheless, sharp peaks are observed at the $\left(5 g ; n l^{\prime}\right)$ energies. The authors argue that Fano's analysis must be modified to explain this result [1].

Section 5 of Fano's paper can be employed in a first approximation to treat the interactions of an excited $\left(5 g ; n l^{\prime}\right)$ state and the $(5 f ; 17,12)$ state from which the oscillator strength derives with the $(5 f ; \epsilon l)$ continuum, taking care to first transform these two discrete states into linear combinations that prediagonalize the bound Hamiltonian matrix, as specified explicitly in Fano's development [2]. In this approximation, Sec. 5 [Eq. (65)] of Fano's paper gives

$$
\begin{aligned}
\sigma\left(E \approx E_{n l^{\prime}}\right) \propto & \frac{\left|\left\langle 5 f ; 17,12|\hat{V}| 5 g ; n l^{\prime}\right\rangle\right|^{2}}{\left(E-E_{5 f ; 17,12}\right)^{2}} \\
& \times\left\{\frac{|\langle 5 d|\hat{\mu}| 5 f\rangle|^{2}\left(\Gamma_{n l^{\prime}} / 2 \pi\right)}{\left(E-E_{n l^{\prime}}-\Delta_{n l^{\prime}}\right)^{2}+\left(\Gamma_{n l^{\prime}} / 2\right)^{2}}\right\}
\end{aligned}
$$

for excitation of an individual $5 g ; n l^{\prime}$ state, where $E=$ $h \nu+E_{5 d ; 17,12}$ and $\Gamma_{n l^{\prime}}$ and $\Delta_{n l^{\prime}}$ are width and shift functions evaluated at the zeroth-order energies $E_{n l^{\prime}}$. This expression predicts Lorentzian lines centered at the shifted $\left(5 g ; n l^{\prime}\right)$ energies whose peak heights are modulated by a slowly varying prefactor, in general accord with the measured spectrum [1]. Of course, more detailed treatments based on additional or alternative discrete and continuum zeroth-order states, perhaps even incorporating the width of the initial state [3], can provide more quantitatively reliable results.

The foregoing expression is also obtained from Sec. 2 [Eq. (16)] of Fano's paper by including a sum over the discrete $(5 f ; n l)$ states in the principal-value integral over background states that occurs in the familiar isolatedresonance formula [2]. Eichmann, Gallagher, and Konik view this as an extension to Fano's treatment, which otherwise ". . .is incorrect for long-range potentials which support bound states..." [1]. However, it has long been understood [4] that Fano's analysis is equivalent to Feshbach partitioning [5], and that the principal-value contribution in the isolated-resonance expression arises from the resolvent of the background Hamiltonian $\hat{P} \hat{H} \hat{P}$, which should include discrete states, if any, in its spectral representation both for closure and to avoid a possible singularity at the ionization threshold in the case of Coulombic potentials.

In summary, two distinct applications of Fano's 1961 formalism [2] employing the zeroth-order basis states of Eichmann, Gallagher, and Konik [1] provide the nonzero cross section indicated above; given the flexibility inherent in Fano's approach, other routes to this result should be possible. Although application of his formalism is usually straightforward, care must be taken when unusual features are encountered in special cases, such as the vanishing of key amplitudes in the application of Eichmann, Gallagher, and Konik [1].

John W. Cooper, ${ }^{1}$ Chris H. Greene, ${ }^{2}$ Peter W. Langhoff, ${ }^{3}$ Anthony F. Starace, ${ }^{4}$ and Carl Winstead ${ }^{5}$

${ }^{1}$ Institute for Physical Science and Technology

University of Maryland

College Park, Maryland 20742-2431, USA

${ }^{2}$ Department of Physics and JILA

University of Colorado

Boulder, Colorado 80309-0440, USA

${ }^{3}$ San Diego Supercomputer Center

University of California

La Jolla, California 92093-0505, USA

${ }^{4}$ Department of Physics and Astronomy

The University of Nebraska

Lincoln, Nebraska 68588-0111, USA

${ }^{5}$ A. A. Noyes Laboratory of Chemical Physics

California Institute of Technology

Pasadena, California 91125, USA

Received 13 August 2004; published 10 June 2005

DOI: 10.1103/PhysRevLett.94.229301

PACS numbers: $32.70 .-n, 32.30 . J c$

[1] U. Eichmann, T. F. Gallagher, and R. M. Konik, Phys. Rev. Lett. 90, 233004 (2003).

[2] U. Fano, Phys. Rev. 124, 1866 (1961).

[3] Y. Komninos and C. A. Nicolaides, Phys. Rev. A 70, 042507 (2004).

[4] B. W. Shore, Rev. Mod. Phys. 39, 439 (1967).

[5] H. Feshbach, Ann. Phys. (N.Y.) 5, 357 (1958); 19, 287 (1962). 